\title{
Le jeu de scène: traductions et traducteurs à travers les cultures et les genres littéraires
}

\author{
Iulia Mihalache \\ Université du Québec en Outaouais (Gatineau, Canada)
}

This article aims at revealing a series of collective and individual representations of translation and of the translating subject present in the work of fiction. The authors chosen for this analysis belong to a multicultural space and are therefore inclined to reflect upon translation. Every author will opt for a specific genre. However, are there any recurrent representations of translation and of the translating subject from one genre to the other, across the cultures and across the different writing styles? In order to analyse the transfer of representations between these genres, but also between translation theory and fiction, we have selected the following texts: Carlos Batista's Bréviaire d'un traducteur (2003), Ólafur Jóhann Ólafsson's Absolution (1994), Dezsó Kosztolányi's Le Traducteur cleptomane (1985), José Carlos Somoza's La caverne des idées (2002), Michel Orcel's Les larmes du traducteur (2001) and John Crowley's The Translator (2002).

\section{Introduction}

La relation entre les textes où le traducteur reste fictif et ceux où le traducteur est aussi un théoricien de la traduction peut être vue comme une relation fondée sur l'analogie, une relation métaphorique capable d'engendrer ou de nourrir des représentations du sujet traduisant, à même de former et de perpétuer des pratiques: "Produire une métaphore revient essentiellement à produire un nouveau langage, ou plus exactement une nouvelle pratique langagière" (Jongen 1980: 116). Le traducteur fictif pourrait alors être considéré comme 'une greffe sémantique' du théoricien de la traduction ou, sinon, comme une voix qui anticipe sur celle de la théorie. Pour interpréter les images du 'sujet traduisant' dans les textes de fiction, nous mettrons ces images en correspondance avec d'autres représentations, stéréotypes, modèles, idées ou idéaux qui circulent à travers les textes théoriques, afin de donner une continuité aux différents 'interprétants' qui montrent le traducteur comme sujet d'un système de rapports linguistiques et culturels, comme voix historique.

Dans le développement de la théorie de l'organisation en structure (structure mapping theory), qui est une théorie du traitement des informations analogues, Gentner (1983) considère la métaphore comme une stratégie cognitive utilisée pour mettre en relation deux domaines de connaissances et pour transférer des connaissances (ou des modèles mentaux) d'un domaine d'origine vers un domaine cible. Les idées de migration entre les domaines (qui, dans notre cas, pourraient être ceux de la fiction et de la tra- 
ductologie) et de 'transfert' des connaissances (et par là de changement des pratiques) sont au cœur de la traductologie contemporaine, qui ne voit plus la traduction comme fondée sur l'équivalence, mais plutôt sur l'analogie, capable de produire de nouvelles représentations, de nouveaux "mèmes de la traduction" (Chesterman 1997), compris comme des formes de savoir objectif (idées reçues) qui se développent, se propagent et transforment le monde mental du sujet traduisant ou du sujet lecteur.

L'analogie crée une interaction entre des objets cognitifs: ceux-ci se trouvent des 'traits' communs, se transforment et modifient ainsi les perceptions que l'on avait d'eux: "L'idée centrale de la théorie de l'interaction est de considérer qu'élaborer et comprendre une métaphore ou une analogie, implique en fait un changement de représentation de l'objet de la description" (Gineste \& Indurkhya 1993: 166). Dans cette optique, peut-on dire que les perceptions véhiculées par les textes de fiction transforment ou orientent les idées théoriques (ou les actions des théoriciens de la traduction) ou encore se créent suite aux représentations que ces textes font circuler, en fonction du contexte culturel qui les accueille? Ou bien que les représentations fictives et théoriques sont dans une interaction continue qui modifie nos perceptions sur ce que sont le traducteur et la traduction? Tout comme les métaphores articulent les pensées, représentent des modèles mentaux ${ }^{1}$ et orientent nos actions, les représentations diverses du sujet traduisant fictif et réel et de la traduction pourraient avoir une incidence non seulement sur la communication en général (le transfert 'actif' d'informations entre les cultures), mais aussi sur la communication entre les disciplines d'une part, entre le traducteur et ses destinataires d'autre part: "metaphors might be useful as indicators and maybe even as predictors of the quality and evolution of the therapist-client relationship", lit-on sur le rôle des métaphores dans la psychothérapie (Moser 2000).

Pour analyser le transfert des représentations du traducteur et de la traduction entre la fiction et la théorie, nous avons retenu plusieurs textes appartenant à des genres différents: les aphorismes de Carlos Batista dans Bréviaire d'un traducteur (2003), l'histoire de la traduction du manuscrit de Peter Peterson, relatée par Ólafur Jóhann Ólafsson dans Absolution (1994), l'histoire du Traducteur cleptomane de Dezsó Kosztolányi (traduit pour la première fois en français en 1985), ${ }^{2}$ le roman philosophique à teinte policière La Caverne des idées de José Carlos Somoza (2002), le journal de Michel Orcel, Les Larmes du traducteur (2001) et les souvenirs de la traductrice fictive Christa Malone, dans le livre The Translator de John Crowley (2002).

\section{Le Bréviaire d'un traducteur comme métacognition}

Les aphorismes de Batista, regroupés sous quatre thèmes représentatifs dans l'histoire de la traduction (L'Art d'aimer, L'Art de trahir, L'Art de séduire et L'Art de fuir), reflètent et répètent différentes représentations ou stéréotypes 
de la traduction et du traducteur. Plusieurs d'entre eux peuvent être placés sous le signe des divers 'mèmes' de la traduction dont parle Chesterman (1997): "les mots", "la parole de Dieu", "la rhétorique", "le logos", "la linguistique", "la communication", "la culture cible" ou "la cognition". Fondés sur la comparaison et l'analogie, les énoncés de Batista sont aussi des outils qui expriment des paradoxes, des contradictions, qui remettent en cause des représentations (rupture) et ouvrent l'espace des idées (innovation). Pour le lecteur traducteur, ce sont des exercices herméneutiques qui amènent ce premier à faire un lien avec la pensée traductive et traductologique. Ce sont des formes de langage qui, malgré qu'elles semblent effacer le sujet auctorial (tout en préservant, par l'emploi du présent, les marques de la subjectivité), demandent au lecteur d'établir des rapports entre les connaissances, d'interpréter et de dialoguer avec l'auteur 'chiffreur'.

La métaphore directionnelle source-cible, qui exprime l'idée d'un texte d'arrivée vu comme une interprétation/représentation d'un texte de départ et imprègne à la traduction le sens du mouvement, du transfert, de la projection et de la diffusion des idées, se révèle chez Batista dans les images de la traduction qui "relève d'un trafic", des "frontières" entre les mots, du "transtextuel" ou encore du "texte immigré".

L'idée d'épidémie des croyances et de contamination des idées, que l'on retrouve chez Sperber (1996), apparaît chez Batista dans l'image d'un traducteur "hanté" par le texte source ${ }^{3}$ (Batista parle aussi du "traducteur chasseur"), dans la référence aux traducteurs “contaminés" par l'original ${ }^{4}$ ou encore dans l'idée d'une dépossession mentale qu'opère l'acte traductif ("Centre d'études des traducteurs aliénés"). La perte de soi (qui peut être aussi un dédoublement: le traducteur devient auteur) présuppose un conflit entre le transfert de l'original et le contre-transfert du sujet traduisant inscrit dans un espace-temps, ${ }^{5}$ ce conflit pouvant aller jusqu'à une violence textuelle réciproque ou au "texticide" (Batista). D'un côté, le transfert des idées de l'original exprime la projection de la culture d'origine, son identification et son interprétation à travers un imaginaire cible existant. De l'autre, le contre-transfert du sujet traduisant est vu comme une réaction aux idées propagées et comme une transformation de l'inconscient du sujet sous l'effet de ces idées.

Nous avons également remarqué la présence de l'idée de 'hantise' dans le roman de l'écrivain islandais Ólafur Jóhann Ólafsson, Absolution (1991), ${ }^{6}$ qui raconte la vie de Peter Peterson, torturé par le cauchemar récurrent d'un crime passionnel qu'il aurait commis cinquante ans plus tôt par dépit amoureux. Après sa mort, les mémoires de Peterson seront traduits par un compatriote, qui se laissera imprégner par la subjectivité de son personnage, doué d'un sens de manipulation aigu, paranoïaque, cynique et orgueilleux. "Please do not let him invade my mind", écrira-t-il (1991: 253).

L'identification du traducteur à la personnalité de son personnage, qui vit dans un monde vide et froid, rempli d'images effrayantes, peut aussi exprimer ce stéréotype du traducteur enfermé dans sa tour d'ivoire ou obligé de mener son existence dans un espace cubique: "Since there are now 
windows in my part of the office, I had gotten up and strolled down the corridor to watch the spring rain as I mulled over a handful of words that needed to be improved in my translation" (Ólafsson 1991: 10; nous soulignons). L'obsession du traducteur d'entrer dans le vécu "froid", sans passion, de Peter Peterson peut aussi indiquer un désir d'objectivité scientifique en traduction: "When I returned home that evening, I began, as if by instinct, to translate the manuscript. I became so obsessed with it that I found myself revising, amending and abridging it as if I were its original author" (Ólafsson 1991: 12; nous soulignons).

Les références à la psychanalyse ne manquent pas dans le texte de Batista, qui évoque le "traducteur fou" ou "paranoïaque" (Batista 2003: 48), la "trahison" (avec ses extensions du "double" et du "paraître") ou le rêvefiction-réalité. Cette triade renvoie à ces représentations sources et cibles silencieuses, qui n'existent qu'à l'état de "mode du reste" (concept emprunté à Lecercle par Venuti [2002]), et qui peuvent être activées dans l'acte d'écriture ou de traduction, ou lorsque le traducteur 'prend en charge' le texte qu'il traduira, et s'y identifie (nous reviendrons sur cet aspect dans l'analyse du livre La Caverne des idées). Dans ce type de traduction, le sujet traduisant entrerait non seulement cognitivement, mais aussi par l'émotion dans le monde narratif qu'il est en train de traduire. Cette approche 'somatique' de la traduction permettrait d'expliquer, selon Robinson (1990), que la traduction n'est ni un processus fondamentalement cognitif, gouverné systématiquement par un ensemble de normes et de structures abstraites, ni un processus intuitif qui ne peut pas être expliqué. La traduction serait plutôt un exercice raisonné durant lequel les décisions du traducteur peuvent être aussi des retours libérateurs de représentations jusque-là refoulées, des formes de résistance à une programmation idéosomatique.

Les aphorismes de Batista qui représentent la traduction comme une 'spiriterie' et le sujet traduisant comme un exorciseur, un médium (Batista 2003: 57), un théologien, ou comme un (al)chimiste abordent la pratique traductive par le prisme des tabous, des répressions et des interdictions. Ce n'est pas pour rien que Batista appelle son livre bréviaire d'un traducteur: il y voit ironiquement un livre indispensable pour le traducteur, collectivement perçu comme quelqu'un qui 'récite' les paroles des autres. D'un côté, la pratique du traducteur semble être contrôlée par des normes et des courants de pensée dominants (Robinson 1996). De l'autre, le sujet traduisant voudrait se situer en dehors de ces idées "fixes" qui entourent sa pratique: produire une traduction fidèle ou une traduction élégante ou encore une traduction littérale, adorer l'original (Batista 2003: 19), voir dans la traduction une sorte d' "éponge", de "filtre" ou de "tamis" (Batista 2003: 42). Tel que le remarque Robinson (1996: 28), une bonne partie des théories de la traduction sont dominées par des "tabous", par un désir de "régulariser" la traduction et de lui attribuer des étiquettes, en laissant de côté la vraie expérience des traducteurs et des lecteurs en leurs qualités d'êtres sociaux.

D'autres mythes de la traduction et d'autres représentations du traducteur se retrouvent dans le texte de Batista, comme celui de l'équivalence, 
de la liberté en traduction et de la traduction comme un acte de réécriture annoncé, préparé par d'autres textes dans les cultures source et cible (ce que Torop [1997: 27] appelle la "traduction métatextuelle"). L'idée de mainmise sur l'original" nous renvoie à la métaphore de la "traduction cannibale" (Vieira 1994), qui imagine que la traduction peut absorber la valeur de l'original et nourrir la langue et la culture cibles pour produire quelque chose de nouveau. À l'opposé du "traducteur cannibale" se trouve celui que Batista appelle le "traducteur diététicien", qui ne valoriserait pas la différence en traduction, mais la laisserait au contraire déperir. On retrouve aussi le mythe de l'intraduisibilité et ses différentes variantes (la traduction parfaite est inaccessible, la poésie est intraduisible), avec une référence à l'impossibilité de traduire le poème "Voyelles" d'Arthur Rimbaud en une langue asiatique; par ailleurs, ce "mème de la traduction" (Chesterman 1997) a fait aussi l'objet d'interrogations dans des domaines comme la localisation ou la traduction assistée par ordinateur (comment prendre en charge, techniquement parlant, toutes les langues de la planète?). Cela ne nous étonne donc pas de voir que, dans l'analyse de la traduction en portugais de ce même poème, Ketchum établit un lien entre la cognition, les perceptions visuelles et la traduction assistée par ordinateur:

It is an example of a very visual, computer-aided translation, that consists of seven arched couplets, each a different color with "Rimbaud" written down one side and "Rainbow" down the other. The importance of color is stressed in the printed version of this translation because, in Rimbaud's sonnet, each vowel sound is related to a color. (Ketchum 2000/2001: 252)

Alors que certains aphorismes de Batista reprennent les idées de 'traductioncopie' et de 'traducteur-serviteur' ou 'invisible' (transparence, soumission, "traducteur marionnettiste" ou marionnette linguistique, traducteur effacé), d'autres tracent le portrait du traducteur communicateur et médiateur entre les cultures ("traducteur ramoneur", "traducteur aviateur", "traducteur maçon" - et la migration se poursuit anecdotiquement jusque sur la planète rouge: "traducteur martien") et révèlent, avec humour, le danger des "culturèmes" . ${ }^{8}$ La communication peut toutefois être subversive: Batista parle alors du "traducteur terroriste", cette image du traducteur situé dans un espace à l'intérieur duquel se déploie, se distribue, se dispute et s'échange le pouvoir social s'inscrivant dans une sorte d'extrême du "virage du pouvoir" (power turn) pris par les études de la traduction selon Tymoczko \& Gentzler (2002). ${ }^{9}$ Batista n'oublie pas non plus les autres activités langagières, comme la révision (Batista 2003: 38) et l'édition et résume en un aphorisme la multiplicité des représentations de la traduction à travers 1 'histoire,$^{10}$ ces "interprétants" assurant la continuité de la pratique traductive.

Carlos Batista, lui-même un traducteur, se fait l'écho de Robinson (1999: 403) quand ce dernier affirme que depuis des siècles la théorie de la traduction se place sous le signe d'une "éthique anecdotique" et que certains théoriciens, en faveur d'une rhétorique scientifique, systématique et dépersonnalisée, envisagent l'anecdote comme un obstacle et une limitation dans 
le progrès de la discipline. Cependant, Robinson conclut à la complémentarité des deux approches, car les anecdotes, les histoires apparemment triviales peuvent avoir, par leur caractère local et par une conscientisation de la pratique, une visée théorique, celle de remettre en question les paradigmes théoriques universels ou ce que Robinson (1996) appelait les pratiques traductives contrôlées par les pensées dominantes:

Anecdotes assume explanatory power in theoretical works by exploiting the intrinsic complexity of local, individual experience in ways that complicate or problematize established theoretical assumptions, norms or propositions and thus advance the field. [...] an anecdote without systematic awareness or reflection becomes dumb. (Robinson 1999: 404, 407)

Batista n' oublie pas non plus le "traducteur infidèle" (par exemple, celui qui 'surtraduit' $)^{11}$ ou encore le "traducteur cleptomane". La loyauté est d'ailleurs un débat central de la traductologie (Robinson 1990; Venuti 1992, 1998; Pym 1997, 2001), avec des racines mythiques et des manifestations diverses (de la fidélité du traducteur antique au traducteur exprimant la différence culturelle à l'époque du Romantisme, et jusqu'au traducteur contemporain préoccupé par la pluralité de ses choix et leurs implications pour sa propre culture [Koskinen 2000]).

\section{Le 'commerce' des idées: le traducteur 'sans éthique'}

L'histoire du "traducteur cleptomane", une autre anecdote, appartient en fait au Hongrois Kosztolányi qui imagine un traducteur dont les idées ne peuvent pas être séparées du désir de voler, de la soif matérielle et donc du commerce. Dans le récit de Kosztolányi, deux amis se rencontrent et commencent à parler du traducteur Gallus - celui qui a un défaut fatal (il "vole comme une pie") et ne sait rien faire d'autre qu'écrire (c'est l'image d'un 'traducteur damné') -, après avoir accepté amèrement l'idée que la gloire - peut-être celle de l'auteur ou encore celle d'une "traduction unique" - est éphémère: "Qui se souvient encore de...? [...] Dans ce silence, la couronne desséchée de sa gloire craquelait comme les feuilles mortes dans un cimetière" (Kosztolányi 1985: 7).

Sorti de la prison après deux ans, le traducteur Gallus rencontre le narrateur et le supplie de l'aider, car "il n'avait pas de travail" (une deuxième image apparaît: celle du 'traducteur pauvre'). On lui confie alors la traduction d'un livre "bon pour la poubelle" que Gallus traduira "avec zèle" et rapidement (la troisième représentation est celle d'un traducteur 'bon à tout faire', qui aime son métier, mais qui est par contre obligé de traduire des livres dont personne ne se souviendra plus tard; pour l'éditeur qui lui confie le manuscrit, l'acte de traduction subit ainsi une marchandisation). Gallus n'est pas seulement un traducteur talentueux, mais aussi un 'bon' éditeur: "Notre ami l'avait joliment dactylographié, avait numéroté les pages et les avait même attachées avec un ruban aux couleurs nationales" (Kosztolányi 1985: 
9; nous soulignons). Le narrateur ne peut d'ailleurs pas s'empêcher de porter un jugement de valeur sur la traduction et il s'exclame au moment de la lecture du manuscrit: "J'ai commencé à lire le texte. Avec des cris de ravissement. Des phrases claires, des tournures ingénieuses, de spirituelles trouvailles linguistiques se succédaient, dont cette camelote n'était peut-être même pas digne" (10).

Ahuri par le mécontentement de l'éditeur, le narrateur se met à 'comparer' la traduction de Gallus avec cet original qu'il considérait comme étant une "camelote". La comparaison, qui s'opère par 'segmentation' du texte original, ne se fait pas pour révéler ce qui a motivé les choix du traducteur, mais pour emprisonner ce dernier dans les idées de l'original:

La première phrase de l'original disait ceci: L'antique château rescapé de tant d'orages resplendissait de toutes ses trente-six fenêtres. Là-haut, au premier étage, dans la salle de bal, quatre lustres de cristal prodiguaient une orgie de lumière ... La traduction hongroise disait: "L'antique château rescapé de tant d'orages resplendissait de toutes ses douze fenêtres. Là-haut, au premier étage, dans la salle de bal, deux lustres de cristal prodiguaient leur orgie de la lumière..." J'ai ouvert de grands yeux et j'ai continué ma lecture. (11)

Les conclusions du narrateur sont accusatrices envers un traducteur 'sans éthique', infidèle, voire inconscient, et elles révèlent aussi la perception que le narrateur a de l'original, même quand ce dernier n'a pas de valeur 'littéraire'. C'est en fait une perception collective qu'il a intériorisée: "En quelque lieu que sa plume ait passé, le traducteur avait causé préjudice aux personnages, et ça à peine connaissance faite, et sans égard pour aucun bien, mobilier ou immobilier, il avait porté atteinte au caractère incontestable, quasi sacré, de la propriété privée" (12). Les mérites du traducteur Gallus sont encore rabaissés quand le narrateur explique:

Pour moi, en tout cas, ce qui m'a paru le plus accablant - car c'était nettement une preuve de mauvaise foi et de veulerie -, c'est qu'il lui arrivait fréquemment d'échanger des métaux nobles et les pierres précieuses contre des matières viles et sans valeur, le platine contre du fer-blanc, l'or contre du cuivre, le vrai diamant contre du faux ou contre de la verroterie. (12)

Finalement, le narrateur évoquera une autre représentation collective de ce qu'est le traducteur, celle d'une subjectivité 'anthropophage'. À ce sujet, il n'est peut-être pas inutile de noter que la perspective du "Mouvement anthropophage" brésilien sur la relation avec une altérité qui s'imagine au centre a eu des échos en traductologie, grâce à Vieira, selon qui "translated texts and colonized cultures, both marginal spaces and, conventionally, considered derivative, tend to be evaluated by what they fail to be in relation to the originating text or culture rather than by what they are" (cité dans Ketchum 2001/2002: 252). N'oublions pas que l'histoire inventée par Kosztolányi a été écrite en hongrois en 1930 et traduite pour la première fois en français en 1985: 
Où les avait-il mis, ces biens mobiliers et immobiliers, qui n'existaient tout de même que sur le papier, dans l'empire de l'imagination, et quel était son but en les volant? Un tel examen nous entraînerait loin, aussi n'irai-je pas plus avant. Mais tout ça m'avait convaincu qu'il était toujours l'esclave de sa passion coupable ou de sa maladie, et qu'il n'y avait pour lui aucun espoir de guérison et que, de la société des honnêtes gens, il ne méritait pas le soutien. (13)

\section{Rechercher la consonance avec l'auteur: le périple de la traduction}

Le journal de Michel Orcel, Les Larmes du traducteur (2001) fait réapparâ̂tre certaines images de la pratique traductive, du texte traduit ou du traducteur. S'agissant d'un journal, on s'attend alors à un épanouissement de la subjectivité. D'ailleurs, Orcel parle d'un journal 'hybride', à la fois pour signifier le mariage entre sa pensée, sa subjectivité et celle de l'auteur qu'il traduit ainsi que son rôle de médiateur, et pour nourrir son expérience de “traduction d'une épopée italienne du XVI e siècle" (Orcel 2001: 7) de 1'observation quotidienne de la vie au Maroc. Le séjour à l'étranger prédispose le traducteur à réfléchir sur la migration des idées et au passage d'une culture à une autre. La traduction apparaît de la sorte comme une initiation: "Je traduis, j'accompagne les mots d'une langue à une autre, et leurs sens volubiles. Je suis le lieu incertain du traduire. Je traverse à petits pas le fleuve roulant de l'arabe. J'aime et je traduis" (11).

Pour Orcel, la rencontre avec le texte original stimule son imagination, c'est une sorte de rêve: "Traduire, comme voyager, exige qu' on soit tout yeux perméable jusqu'à l'illusion de se perdre dans l'objet - mais en allant chercher au fond de soi le visage fantastique du récit" (28). Mais ce rêve, demeure des représentations réprimées, silence qui précède le texte traduit, suppose une transformation violente et un renouveau: "la violence du traduire", affirme le traducteur (17). Toutefois, le traducteur retournera toujours à lui-même - "Fuyant sa langue sans répit, sans cesse y faisant retour" (64) - après avoir parcouru, à son allure, le même chemin que son auteur (68) et après avoir "vérifié la validité de [ses] choix" (34).

\section{Dialoguer sur la rive: l'émergence de l'identité traductive}

Situé en 1960, dans le contexte de la guerre froide, le roman The Translator (2002) de John Crowley raconte l'histoire de la jeune Christa (Kit) Malone, élève et traductrice du poète russe Innokenti Isayevich Falin exilé aux ÉtatsUnis. C'est un roman d'espionnage situé 'entre deux mondes' (la Russie et les États-Unis), mais aussi un livre d'amour et une collection de souvenirs de la traductrice Christa Malone. Le mystère flotte tout au long du roman, non seulement à cause de la tension et de l'ambiguïté dans lesquelles vit le poète russe exilé aux États-Unis ("un état de traduction continue") ${ }^{12}$ - celui qui affirme être un enfant sans identité et amour (un besprizornyi) -, mais aussi 
à cause du mystère qui caractérise la relation entre la jeune Christa et son professeur Falin, deux êtres qui vivront toujours "sur la frontière" (frontières entre les langues, entre les pays, entre les âges). Ce lien flou évoque aussi la crainte d'un espace géographique 'autre', lointain, innommé, imaginé comme étant primitif, et pour cette raison dangereux:

When she was a child, and for a long time afterward, Kit Malone always imagined Russia as dark. It was dark then; a Dark Continent from which no real news came, a dark star absorbing its own light. When she thought of it she saw roads leading into the hinterlands, cold featureless steppe without colour or sound, and huddled peaple silent too, their backs turned to her. (Crowley 2002: 7)

Kit Malone rencontre Falin à l'Université quand elle n'a que 19 ans. Dès le début, elle est étonnée par la capacité de Falin de "se rendre inaperçu", de rester 'invisible'. Le 'jeu' entre l'auteur et sa future traductrice commence lorsqu'il lui donne sa première leçon: "I asked him how he comes by this ability to appear and disappear, and he told me it was easy, and I could do it too: I needed only to practice invisibility, as he had" (Crowley 2002: 21). Tout au long du roman, Crowley montre comment la future traductrice part de l'état d'humilité du personnage qui vit dans l'ombre, qui file son auteur comme un 'espion' ${ }^{13}$ et comment elle parcourt 'volontiers' le même chemin que son auteur pour finalement se dévoiler et produire une œuvre dont on ne devinera plus ses intentions d'origine: “'Translations without originals' she had called them: poems neither his nor hers, or both his and hers; poems written in a language that she couldn't read, and surviving only in a language he couldn't write" (8).

Falin enseigne la poésie dans une université américaine et apprend aux étudiants que la poésie est intraduisible quand on ne voit que les mots. À une époque où les langues sont considérées comme des codes et où la traduction est associée, pour des raisons politiques, à un déchiffrement, dans un monde trouble où la parole peut avoir des conséquences sociales, le poète russe explique la nécessité de son dédoublement: “'I knew,' he said. 'I knew. Not to run; to listen. To be not seen when looked at; to be seen when I chose; to speak, to agree, to seem" (Crowley 2002: 132). Auteur et traducteur à la fois (traducteur de l'espace où il vit), Falin souligne l'importance du rôle du traducteur dans la société, mais aussi l'indissociabilité de l'acte d'écriture et de traduction des faits quotidiens de la vie. ${ }^{14}$ Pour reprendre les paroles de Theo Hermans (1996): “Translators never 'just translate'. They translate in the context of certain conceptions of and expectations about translation. [...] Translators too are social agents".

Pour Falin, le traducteur doit toujours lire la pensée de l'auteur qu'il traduit, se laisser 'contaminer'. Toutefois, cette contamination est réciproque et le poète russe semble vivre avec cette crainte de la 'contagion des idées' à la fois dans sa vie réelle et dans sa vie 'fictive': "One fear only he had, I think: that they would touch him, soil him - that they would find somehow means to make him one of them" (Crowley 2002: 21). Après le cours de poé- 
sie avec Falin, Kit décide d'apprendre la langue de celui qu'elle aimait en silence et suit un cours intensif de russe pendant l'été. Elle devient, pour reprendre l'aphorisme de Batista, le "traducteur chasseur", une sorte de Diane, déesse farouche et éternellement jeune. C'est ce même été qu'elle visite Falin, lequel lui propose alors de l'aider à traduire ses poèmes, sans “détourner" la forme et l'harmonie de sa pensée: "“No! No no. You will not march all over my poems with muddy footnotes"" (181). Le dialogue entre Christa et son professeur, avant d'entamer la première traduction, révèle deux mythes de la pratique traductive - la traduction 'linguistique' et la traduction 'stylistique': "'I can't do that,' she said. 'I don't know this language at all. You said so yourself.' 'But you can hear it. You can hear the meanings, which are part of the music. And you have English music"' (173).

L'appréhension de l'altérité russe séduira Christa $^{15}$ et la transposera dans une sorte de rêve magique où la traductrice doutera de ses actes et de son identité (l'image du rêve revient chez Crowley, également, et Christa ne se rappellera plus si Falin a été aussi son amant). La découverte des idées de l'autre est associée à la découverte du corps d'un amant ou à la recherche de la perfection et de l'unité (209). La traductrice entre émotionnellement et cognitivement dans l'espace de son auteur et l'interprète, tout en sachant que ces interprétations sont multiples: ${ }^{16}$

She thought, long after, that she had not then ever explored a lover's body, learned its folds and articulations, muscle under skin, bone under muscle, but that this was really most like that: this slow probing and working in his language, taking it in or taking hold of it; his words, his life, in her heart, in her mouth too. (183)

Après la traduction, suivra la possibilité de 'trahison', qui sera double dans le roman: trahison par la traduction et trahison dans la vie réelle, car on demandera à Christa d'espionner Falin, soupçonné de collaborer avec les Russes. La crainte de la 'contamination' obligera Falin de s'éloigner de sa traductrice, sans pour autant la quitter réellement dans sa pensée (Christa aura toujours l'impression d'être poursuivie): "'Kit,' he said. 'I must say this now. Not easy to say. It has become dangerous that you should be nearby me. You must from now on stay away"' (Crowley 2002: 229). La disparition de Falin sera aussi ambiguë que ses propres origines (on diffusera l'idée qu'il s'est noyé volontairement ou qu'il a peut-être été tué) et cette disparition pourra, comme tout texte qu'on lit, traduit ou que l'on transpose d'une culture à l'autre, générer une suite d'interprétations diverses. Pour comprendre les significations d'un autre monde, Christa voyagera en Russie, où elle rencontrera ceux qui avaient connu Falin:

'Kit,' he said. He sat by her. 'Listen. Tomorrow, later on, they may say they know what became of me, what happened, but they will be wrong. Because an act - any act - may be one thing in one world and something else in another world, a thing that is not like it but has its shape, that rhymes with it. A commonplace thing, accident or loss, it may mean nothing here and everything there...' (255) 


\section{Créer et se placer dans un état continu de demande interprétative: l'exercice herméneutique de la traduction}

L'hybridité du texte, comme aussi la pluralité de voix qui forment le discours sur ce qu'est la traduction ("the untidiness of our disciplinary translation of translation" [Hermans 1996]), incite le lecteur et le traducteur à intervenir dans le texte, à agir "not only upon the text but within it, engaging in rivalries with the various voices in the text, as well as with the author, publisher and reader outside the texte" (May 1994: 6). Le roman La Caverne des idées (2002) de Somoza, dont l'intrigue est digne d'Umberto Eco, s'inscrit dans ce que Steiner (1975: 48) appelle "understanding as translation", mais aussi dans un modèle herméneutique (Hermans 1996) où la traduction devient à la fois un acte de transmission et de récupération culturelles - à même de rendre intelligible, à travers la voix du traducteur, le message des dieux -, et un acte d'interprétation et de représentation. Car le récit du 'Déchiffreur d'Énigmes', Héraclès Pontor, chargé par le mentor de l'Académie de Platon, Diagoras, d'enquêter sur la mort d'un jeune éphèbe trouvé mort dans les rues d'Athènes (supposément attaqué par des loups), est en fait le récit d'une expérience de traduction qui se greffe sur l'histoire principale du roman, et dont le but est de retrouver les 'vraies' significations ou la 'version officielle' d'un événement. Un traducteur anonyme traduit un ancien manuscrit et le retranscrit sous nos yeux, convaincu que le texte contient une clé de lecture. L'auteur aurait délibérément enlevé certains passages ou déchiré des bouts de textes pour compliquer la lecture et obscurcir les faits:

Les cinq premières lignes sont manquantes. Dans son édition du texte original, Montalo affirme que le papyrus a été déchiré à cet endroit. Je commence ma traduction de La Caverne des idées à la première phrase du texte de Montalo, qui est le seul que nous ayons à notre disposition. (N.d.T.) (Somoza 2002: 11)

Il accompagne sa traduction d'une réflexion non seulement sur le texte et sur ses décisions de traduction (le texte est parsemé des notes en bas de page du traducteur), mais aussi sur sa propre vie, donnant ainsi l'impression de dialoguer avec les personnages ou de dialoguer avec l'auteur du texte (dans cette optique, le roman de Somoza s'inscrit aussi dans ce que Robinson [1990] a appelé la "traduction dialogique", tandis que le traducteur devient "réversionnaire"). ${ }^{17}$ La lecture et la traduction sont guidées par la conviction du traducteur que la technique littéraire appelée eidesis constituait la stratégie de l'auteur du texte. Cette stratégie, qui permettrait de dissimuler un message secret au sein d'une œuvre par le biais d'une récurrence de significations qui partagent des sèmes communs (ce que Greimas appelait des isotopies) et de métaphores, n'avait pas été comprise par Montalo, dans son édition savante de l'original:

L'abus de métaphores liées aux "mèches" ou aux "chevelures", dispersées ici et là, attire l'attention depuis le début du texte: il est possible qu'elles signa- 
lent la présence d'une eidesis, mais cela n'est pas encore sûr. Montalo ne semble pas l'avoir remarqué, car il n'en fait pas mention dans ses notes. (N.d.T.) (Somoza 2002: 15)

Le traducteur, comme celui d'Ólafsson (1991) engagée dans la traduction du manuscrit de Peter Peterson, devient hanté par la répétition de métaphores qui pourraient non seulement 'mener à la vérité du texte original', mais aussi - et c'est ce qui est plus intéressant - "former une idée ou une image indépendante du texte originel" (Somoza 2002: 24), produire de nouvelles représentations:

Indépendamment de leur finalité à l'intérieur de la fiction du dialogue, dans ces dernières phrases: "Il y a toujours des idées au-delà des mots" ... "Et elles sont la seule chose importante", je vois en même temps un message de l'auteur afin de souligner la présence d'une eidesis. Montalo semble, comme toujours, ne rien avoir remarqué. (33)

L'obsession du traducteur persiste jusqu'à ce qu'il découvre, à l'intérieur du texte qu'il est en train de traduire, des messages qui lui sont adressés et jusqu'à ce qu'il soit kidnappé par un inconnu qui le presse de terminer sa traduction, de 'livrer le message'. À ce moment, l'histoire réelle se confond avec la traduction et avec la vie du traducteur anonyme qui se sent menacé par le texte original et responsable de ces actes.

Dans sa traduction commentée, le traducteur anonyme organise, en tant que sujet conscient, les expériences et donne un sens, une forme, une représentation au monde. Il vise à appréhender le texte dans sa "forme-sens", dans son "homogénéité du dire et du vivre" (Meschonnic 1970: 144) et il se sent responsable de son interprétation. Le monde n'existe alors que par rapport au sujet ou par rapport à sa perception (Mannheim 1954). Le traducteur s'implique dans son texte en y transférant ses propres idées et attentes, ce qui met en contradiction ce qu'il croit avec une certaine intentio auctoris et avec ce qui pourrait être établi comme scientifiquement correct. Selon Meschonnic (1970: 41), "la valeur-œuvre ne vit que du conflit entre la nécessité intérieure du message individuel (qui est créativité) et le code (genre, langage littéraire d'une époque, etc.), commun à une société ou à un groupe". Le traducteur fait sortir son interlocuteur d'une certaine lecture canonique d'un texte et enseigne au lecteur qu'il existe, dans le texte, une 'voix du traducteur':

[...] confusing a listener or a reader who (you think) is too locked into a rigid, habitual conception of the world is a way of pushing that person out onto the road to understanding, a way of shaking him or her awake, smashing the easy habit of ideosomatic knowing. (Robinson 1990: 238)

L'expérience de traduction et, par là, de production de représentations est aussi une exploration du processus métaphorique, car toute métaphore constituerait, selon Aristote dans la Rhétorique, une énigme. Le roman explore à la fois la translatio et le translatum, en essayant d'expliquer comment le 
sujet humain construit et manipule des modèles mentaux dans le but de chercher des alternatives ou des conclusions plausibles: "Lorsqu' on analyse une métaphore, on confond souvent ce que la rhétorique latine appelle translatio, c'est-à-dire le processus sur lequel se fonde la métaphore, et le translatum, c'est-à-dire le résultat de ce processus" (Gardes-Tamine 2003). Le traducteur anonyme est présent dans le texte, en l'attente d'une interprétation. Cette interprétation se développe quand le traducteur trouve des zones de sens connues du lecteur (son interlocuteur) et de lui-même, mais aussi quand le traducteur réussit à se transposer dans le monde narratif du texte grâce à son savoir culturel, collectif de ce qu'est l'acte de traduction. Le traducteur charge cognitivement son lecteur, l'exposant à une multitude d'interprétations possibles que seule la fiction, dit le roman de Somoza, pourrait contenir.

\section{Conclusion}

À travers l'analyse des images du traducteur fictif dans des textes appartenant à des genres littéraires différents, nous avons voulu non seulement établir une analogie entre ces représentations et celles qui circulent en traductologie, mais aussi montrer qu'il existe, dans le discours sur la traduction, une pluralité de voix, une hétérogénéité des images du sujet traduisant et de la pratique traductive. Les œuvres analysées, mettant en scène des expériences de traduction, ont des résonances en dehors des textes, dans l'espacetemps qui les a créées, mais aussi dans l'espace de la traductologie où l'œuvre 'objet', issue ou vécue par une conscience (le traducteur) devient à la fois sujet, voix historique et produit d'une pensée collective, d'un système, d'un groupe. Confrontant l'expérience de traduction à la réflexion sur l'acte traductif, c'est-à-dire à une extension de la pratique, nous avons tenté d'appréhender le texte dans sa "forme-sens", dans son "homogénéité du dire et du vivre" (Meschonnic 1970: 144). Les images du traducteur fictif, qui 'marche sur les traces de l'auteur' ou qui, au contraire, se constitue en tant que subjectivité et identité, ont suscité des questions non seulement sur le processus de traduction, la nature du texte traduit et les transformations qu'un texte subit lors de la traduction, mais aussi sur la réception des traductions par les lecteurs et sur l'interchangeabilité des rôles de traducteur et de lecteur. L'acte de lecture, qu'il s'agisse d'une lecture comparative, isolée ou dialogique, favorise la création et le maintien de la 'demande interprétative' et montre que la traduction n'est pas seulement produite, mais lue également, qu'il y a des 'écritures de la traduction' et que la traduction peut aussi devenir une stratégie métacognitive. 


\section{Bibliographie}

\section{Sources primaires}

Batista, Carlos (2003). Bréviaire d'un traducteur. Paris: Arléa.

Crowley, John (2002). The Translator. New York: William Morrow.

Kosztolányi, Dezsó (1985). Le Traducteur cleptomane et autres histoires. Aix-enProvence: Alinéa.

Ólafsson, Ólafur Jóhann (1991). Absolution. New York: Pantheon Books.

Orcel, Michel (2001). Les Larmes du traducteur. Journal du Maroc. Paris: Bernard Grasset.

Somoza, José Carlos (2002). La Caverne des idées (tr. M. Millon). Paris: Actes Sud.

\section{Sources secondaires}

Aristote (2000). Rhétorique (tr. M. Dufour \& A. Wartelle). Paris: Les Belles Lettres. Chesterman, Andrew (1997). Memes of Translation: the Spread of Ideas in Translation Theory. Amsterdam/Philadelphia: Benjamins.

Ehrlich, Marie-France, et al. (éds.) (1993). Les modèles mentaux. Approche cognitive des représentations. Paris: Masson.

Gardes-Tamine, Joëlle (2003). "La métaphore, entre translatio et translatum", 5th International Conference on Researching and Applying Metaphor. On line at: www.info-metaphore.com/articles/gardes-tamine.html.

Gentner, Dedre (1983). "Structure-mapping: A Theoretical Framework for Analogy". Cognitive Science 7, 155-170.

Gineste, M.-D. \& B. Indurkhya (1993). "Modeles mentaux, analogie et cognition". M.-F. Ehrlich et al. (1993), 143-173.

Hermans, Theo (1996). "Translation's Other", Inaugural Lecture delivered at University College London on Tuesday 19 March 1996. On line at: www.ucl.ac.uk/dutch/pages/th.html.

Ingram, Susan (2001). "Translation Studies and Psychoanalytic Transference". TTR 14(1), 95-114.

Johnson-Laird, Philip (1993). "La théorie des modèles mentaux". M.-F. Ehrlich et al. (1993), 1-20.

Jongen, René (éd.) (1980). La Métaphore. Approche pluridisciplinaire. Bruxelles: Facultés Universitaires Saint-Louis.

Ketchum, Nell E. (2001/2002). "Review of: Peter Bush and Kirsten Malmkjær, Rimbaud's Rainbow: Literary Translation in Education". Cadernos de Tradução 8, 250-253. On line at: www.cadernos.ufsc.br/download/8/pdf/Resenhas_Cadernos8.pdf.

Koskinen, Kaisa (2000). Beyond Ambivalence. Postmodernity and the Ethics of Translation. Tampere: University of Tampere.

Mannheim, Karl (1956). Idéologie et utopie. Une introduction à la sociologie de la connaissance. Paris: Librairie Marcel Rivière et Cie.

May, Rachel (1994). The Translator in the Text. On Reading Russian Literature in English. Evanston: Northwestern UP.

Meschonnic, Henri (1970). Pour la poétique. Paris: Gallimard.

Moser, Karin S. (2000). "Metaphor Analysis in Psychology - Method, Theory, and Fields of Application". Forum: Qualitative Social Research 1(2). On line at: http://qualitative-research.net/fqs/.

Robinson, Douglas (1990). The Translator's Turn. Baltimore: John Hopkins UP. 
Robinson, Douglas (1996). Translation and Taboo. DeKalb: Northern Illinois UP. Robinson, Douglas (1999). "Nine Theses About Anecdotalism in the Study of Translation (With Special Reference to Sherry Simon, ed., Culture in Transit)". Meta 44(2), 402-408.

Sperber, Dan (1996). La Contagion des idées: théorie naturaliste de la culture. Paris: O. Jacob.

Steiner, George (1975). After Babel: Aspects of Language and Translation. New York: Oxford UP.

Torop, Peeter (1997). "The Position of Translation in Translation Studies". A. Mauranen \& T. Puurtinen (éds), Translation-Acquisition-Use. Jyvaskylä, Finlande: AFinLa, 23-41.

Tymoczko, Maria \& Edwin Gentzler (éds) (2002). Translation and Power. Amherst: University of Massachusetts Press.

Venuti, Lawrence (2002). "The Difference that Translation Makes: the Translator's Unconscious". A. Riccardi (éd.), Translation Studies. Perspectives on an Emerging Discipline. New York: Cambridge UP, 214-241.

Vieira, Else Ribeiro Pires (1994). "A Postmodern Translation Aesthetics in Brazil". Mary Snell-Hornby (éd.), Translation Studies: An Interdiscipline. Amsterdam: Benjamins, 65-72.

1 "Un modèle mental est une représentation interne d'un état de choses (state of affairs) du monde extérieur" (Johnson-Laird 1993: 1).

2 Kosztolányi fait paraître en 1933 un cycle de plus de quarante nouvelles auquel le personnage de Kornél Esti donne son nom. Ces nouvelles sont parues en français dans trois séries différentes: Le Double, L'œil de mer et Le Traducteur cleptomane.

3 "Le texte étranger crée un besoin chez le traducteur, qui exige inconsciemment que ce texte comble son besoin et qui le modifie en conséquence" (Venuti 2002: 223).

4 'Il n'y a qu'en s'enroulant sur l'original comme un serpent, qu'en s'attachant à lui comme un virus, jour et nuit, sans relâche, qu' on peut avoir l'espoir de réussir sa traduction (et de frôler la dépression)" (Batista 2003: 13).

5 "Un traducteur psychiatre disait: 'Les résistances suscitées par l'original provoquent parfois chez le traducteur une perplexité, un dérèglement semblable à celui qui subjugue le cerveau des amants: obsession tenace, rage meurtrière, prostration, insomnie..."' (Batista 2003: 19).

${ }^{6}$ Ce roman a été couronné par le Icelandic Literary Award et il a été traduit dans plusieurs pays/langues. Nous nous servirons dans ce qui suit de la traduction américaine.

7 "Un traducteur Don Juan disait: 'Dans la langue des Hurons, le mot manger change avec chaque aliment. Y a-t-il une langue où le mot aimer changerait aussi avec chaque conquête?"” (Batista 2003: 18).

8 “'...] le mot serpent a été traduit en eskimo par l'expression tuyau qui mord. On aurait pu dire aussi saucisse qui glisse ou lacet qui siffle" (Batista 2003: 40).

9 "The 'cultural turn' in translation studies has become the 'power turn', with questions of power brought to the fore in discussions of both translation history and strategies for translation" (Tymoczko \& Gentzler 2002: xvi).

10 "Traductions du mot traduction: translation, version, interprétation, adaptation, recréation, transposition, calque, paraphrase, transcodage, translittération, équivalence, conversion, vulgate... Sa définition même n'en finit pas de se traduire..." (Batista 2003: 94).

11 'Un traducteur horloger disait: 'Quand la traduction et l'original s'épousent aussi parfaitement que les deux aiguilles d'une horloge à midi et à minuit, la grosse 
aiguille dit: 'J'aime tes tics', et la grande aiguille surtraduit: 'J'adore tes tacs"”'(Batista 2003: 15).

12 "She had understood all that he had said, with no way of knowing what he meant. It was as though he himself existed here in this town in this state in translation, ambiguous, slightly wrong, too highly colored or wrongly nuanced. Within him was the original, which no one could read" (Crowley 2002: 59).

13 "When she did see him she could often not keep from following him, unseen, shadowing him, which was easier to get away with than she would have thought; all it took was alertness and a heart quiet enough to make the right smooth movements so that the other's peripheral vision was not alerted" (Crowley 2002: 79-80; nous soulignons).

14 "'I point out one example,' he said. 'Where this translation said I will denounce my neighbor my poem said only I will write about my neighbour.'

'Why would they translate it that way then?'

'Because the translator was clever enough to know that in my country now, if we say someone has written about someone else, we mean the person has supplied the authorities information or just speculation, enough perhaps to have him investigated, even arrested. We say of someone, I don't trust her - I think she writes. So the poem may be read in that way, and that is why the translator chose this word denounce. But to write, in Russian, is still also to - to just write. Write letters, poetry.'

She had never tried to translate poetry in any way except literally, as though cracking a code in which is was hidden, a chest or safe more beautiful than what was kept in it" (Crowley 2002: 57).

15 "What she didn't and probably couldn't know about him gripped her, it was a fascination that seemed to her pure, almost impersonal, like a scientist's obsession with the source of a river or the unseen side of the moon" (Crowley 2002: 79).

16 " 'Like poems. You cannot translate. You can only make other poems.' [...] Then what's the original?'

'I don't know. Perhaps there is not one. Perhaps there are many translations but no original"” (Crowley 2002: 181).

17 " [T] he reversionary translator must begin by opening or sensitizing him- or herself to a dialogical conception of the translation: think of yourself in dialogue with the SL writer" (Robinson 1990: 222). 